\title{
The Massachusetts Eye and Ear Infirmary Illustrated Manual of Ophthalmology Mobile Edition Text with PDA Download
}

\author{
Editors: Neil J. Friedman, Peter K. Kaiser and Roberto Pineda \\ 2009, Softcover ISBN: 978-1-4160-6175-5 Saunders Elsevier
}

Jonathan Shahar • Anat Loewenstein

Received: 18 July 2010 /Accepted: 22 July 2010 /Published online: 1 September 2010

(C) Springer-Verlag 2010

The Illustrated Manual of Ophthalmology is a soft-cover manual organized by three principal authors, with additional contributions and reviews by other ophthalmologists from their institute. The authors' goal was to "...produce a concise manual that covers a broad variety of ophthalmic disorders and present it in a user-friendly diagnostic atlas". They are to be congratulated for succeeding in fulfilling that daunting objective.

This book is very easy to read, and meets the needs of ophthalmologists at all levels of training, thus accommodating a broad readership. The photographs and figures that accompany the text are well-chosen in order to ensure better understanding of the subject matter.

Each of the 12 chapters focuses on a single anatomical structure and its different pathologies. The chapter on glaucoma, for instance, deals with glaucomatous optic nerve damage, as well as with other optic disk diseases. This is not the conventional format, but one that has the advantage of guiding the reader in following the steps of an ophthalmological examination, roughly from the anterior to the posterior ocular structures. This makes it both easy and enjoyable to follow the processes.

Most chapters start with a section about trauma injuries of the selected anatomical structure, followed by an introduction to common and less common diagnoses. The presentation of each diagnosis is straight-forward, and free

J. Shahar A A. Loewenstein $(\bowtie)$

Department of Ophthalmology, Tel-Aviv Medical Center,

6 Weitzman Street,

Tel-Aviv 64239, Israel

e-mail: anatlow@netvision.net.il of unnecessary elaborated pathophysiology and commentaries on basic mechanisms. The focus is on clinical findings, differential diagnoses, work-up protocol and treatment planning - all the ingredients that make this book handy in everyday clinical practice.

The spectrum of diagnoses in every chapter is comprehensive, and the main clinical issues for each pathology are discussed and elaborated upon by good quality images.

The depth in which various ophthalmologic diseases are discussed is well-correlated to the extent of how often they are encountered in regular clinical practice. Importantly, the conclusions of clinical studies and epidemiologic data relevant to the discussion are presented as well, and are highlighted.

The data in this text are updated and relevant to current practice.

A special section devoted to treatment planning is added in most disease presentations. The suggestions for therapeutic management include specific medications, dosages and procedures needed for early and late treatment. This part is extremely helpful for a variety of settings, especially that of the emergency room.

The last chapter of this manual gives a short and valuable overview of the technologies available in current ophthalmic practice.

In conclusion, this manual provides answers to everyday clinical questions and gives an interesting and wide overview on general ophthalmology. It is helpful for students, residents, and specialists alike. It is thorough, the language is clear and easy to follow and, no less importantly, it makes for an enjoyable read thanks to the many superb photographs and illustrations. 